\title{
A standardized patient and faculty's viewpoint on working together on an autism spectrum disorder simulation
}

\author{
Constance E. Mclntosh*, Cynthia Thomas, Andrew Edwards \\ School of Nursing, Ball State University, United States
}

\author{
Received: November 29, 2017 \\ Accepted: March 1, 2018 \\ Online Published: March 14, 2018 \\ DOI: $10.5430 /$ jnep.v8n7p91 \\ URL: https://doi.org/10.5430/jnep.v8n7p91
}

\begin{abstract}
Simulated clinical experiences are an alternative to on-site clinical training. Focused simulations allow for teaching, clinical feedback, and adjustments in patient interaction strategies conducted in controlled real-time environments. Simulations allow nursing students to develop skill acquisition, improve critical thinking, problem-solving, and decision making especially when using a standardized patient (SP). The International Organization for Clinical Simulation and Learning defines and SP as an individual specifically educated to depict a patient or person with a particular condition or behavior in a realistic, consistent and repeatable method and educated to consistently represent a patient or person in a scripted situation to instruct, practice, and evaluate. While there is a plethora of information about the participants experience with the simulation process little is known about the SP's personal experience. This paper discusses the standardized patient's experience and the faculty viewpoints after several autism simulations were implemented.
\end{abstract}

Key Words: Simulation, Standardized patient, Nursing education

\section{INTRODUCTION}

Simulated clinical experiences are an alternative to on-site clinical training. These type of focused simulations allow for teaching, clinical feedback, and adjustments in patient interaction strategies to be done in controlled real-time environments; allowing nursing students to develop skill acquisition, improve critical thinking, problem-solving, and decision making when using a standardized patient. ${ }^{[1,2]}$ Simulations have evolved from the use of static mannequins to high fidelity simulators and now have progressed to the use of SP's. ${ }^{[1,3,4]}$ An SP is defined as an individual specifically educated to depict a patient or person with a particular condition or behavior in a realistic, consistent and repeatable method, and a person educated to consistently represent a patient or person in a scripted situation to instruct, practice, and evaluate..$^{[5-8]}$

There is an increasing number of publications exploring the use of an SP with varying degrees of complexity including patients with a single diagnosis to more challenging patients with multiple diagnoses and comorbidities. ${ }^{[1,9]}$ As nursing education moves forward with complex SP scenarios faculty will find it more demanding to create such simulations in part due to recruiting the SP, retaining the SP, continual training and evaluating the SP performance all while ensuring the simulation outcomes are met. ${ }^{[10,11]}$

Although there are a plethora of published articles on the value of nursing simulation, in general, there is a dearth of information regarding the simulation experience from the perspective of the SP. The SP is responsible for bringing the

*Correspondence: Constance E. McIntosh; Email: cemcintosh@bsu.edu; Address: School of Nursing, Ball State University, United States. 
patient to life for the students. However, educators may not have an understanding of the motivation and dedication SP's develop for simulation participation. This article will give a personal account of an SP's experience (in his own words) and how he prepares for each simulation. Furthermore, this paper will also highlight specific areas where the SP and faculty worked together to enhance the simulation by using the SP's expertise to interact with the students.

\section{BACKGROUND}

Benner et al. (2010) ${ }^{[12]}$ advocated exposure to hands-on learning, preferably in a clinical setting. However this active participation learning can also be done in a simulation laboratory. The unique approach of simulation allows for the synthesis to occur between didactic learning and clinical application and may improve critical thinking, at the moment problem solving and real-time communication. ${ }^{[12,13]}$ While there are large amounts of published literature about simulation, they have most often focused on simulation development, outcomes, evaluation, and student learning.

Over time, nurse educators have strategically moved beyond static mannequins to more sophisticated high fidelity simulators but now have found that the use of an SP allows for greater critical thinking, problem-solving, decision making and communication between students and the SP. ${ }^{[9,14,15]}$ Lack of clinical sites, a shortage of patient opportunities for student learning, and the need for students to prepare for the transition into professional practice have created the demand for faculty to develop more realistic simulations. ${ }^{[12]}$

Autism Spectrum Disorder (ASD) presents with a range of conditions characterized by challenges in social skills, repetitive behaviors, and verbal and non-verbal communication. ${ }^{[16]}$ Creating an ASD simulation, that is realistic but maintains safety create opportunities for the faculty. Ethically, it would be inappropriate to use individuals diagnosed with ASD because the simulation itself may evoke negative responses, in the form of emotions (e.g., fear, anger, sadness) or behaviors (e.g., self-injurious, aggression), with the individual. These types of responses could be detrimental for the individual (e.g., physical harm, anxiety-inducing) but also for students (e.g., fear, inability to learn). Students intervening with patients with behavioral issues must learn in a scripted, predictable environment so that the scenario can be controlled to prevent harm to the students. Using individuals with ASD would certainly garner realistic responses, however, could put students at risk because of safety issues (e.g., hitting, biting, slapping). Utilizing an SP, knowledgeable on the diagnosis and common behaviors, is essential in creating an environment where the behaviors are simulated just enough for a student to react to the behavior but not enough to cause harm to the student.

Some schools of nursing use theater majors. However, training or coaching a theater student may prove to be inconsequential due to their lack of knowledge of related symptoms, medications, or diagnosis-related information. Ideally, an SP would already have foundational knowledge; understanding or experience with the specific disorder or diagnosis, medications often prescribed, and treatments - therefore would only need time and help to learn the specific role and simulation script. Tuzer et al. (2016) ${ }^{[17]}$ research indicates the use of standardized patients in simulations were more effective than high fidelity simulators when teaching cardiac, thorax and lung examinations because students could listen to real hearts and breath sounds and exam a real thorax area of the body while communicating with a live individual. To be realistic, the SP must be immersed as the individual being portrayed. To provide the best experience possible for both students and the SP it is important to learn more about how the person "becomes the patient". All simulation participants may benefit from answers to the following questions: How does the SP become immersed in the role? What is the simulation experience like for the SP? How does the SP remain fresh when replaying the role multiple times in consecutive sessions?

\section{THEORY}

The National League for Nursing (NLN) Jeffries Simulation Theory was used during the simulation experiences discussed in this paper. ${ }^{[18]}$ As Jeffries et al. (2015) ${ }^{[18]}$ pointed out every aspect of the simulation process is important to the overall learning and success for participants. We believe this also applies to the SP, as this person is not static but an active participant learning as well. A good simulation design includes detailed learning objectives for participants, and clear objectives should also apply when employing an SP. The SP should be carefully considered as part of the overall experience when designing the simulation to be an interactive and collaborative experience.

The facilitator, SP, and participant share a responsibility to develop an element of trust during the simulation and to fully participate to promote a real experience. ${ }^{[18,19]}$ We agree with Fenske et al.'s (2013) ${ }^{[20]}$ perspective that participants attributes are innate and often come to the simulation experience with anxiety, self-confidence or lack of, and therefore; impact the overall simulation experience. ${ }^{[21]}$ However, this concept also applies to the SP as the SP's age, ethnicity, and gender and knowledge about the subject may determine if the simulation is authentic. The SP as well may be anxious and lack confidence potentially creating an atmosphere of unpreparedness for the role. Jeffries et al. $(2015)^{[18]}$ also 
pointed out that outcomes are not only for participants but newer literature indicates the need to pay attention to the outcomes of the SP or care givers trained through a simulation process. Overall, the SP is a unique individual and plays an important active role in the complete simulation process. The SP should be carefully considered, educated and debriefed to achieve learning outcomes for the participants as well as the SP.

\section{Standardized Patient}

For over a three-year period, faculty at a Midwestern university engaged an individual to serve as an SP for both research studies and program evaluation simulations, specifically ASD. All simulations met with the approval of the University Internal Review Board (IRB). The SP had employment experience working as an Applied Behavioral Assistant with teenagers diagnosed with ASD. He was recruited for the simulation role due to his work related experience, intimate knowledge (e.g., medications, treatment modalities) of ASD, and ability to portray a realistic patient (e.g., specific behaviors).

\section{Pre-Post simulation}

Faculty created simulations about a 14-year old male diagnosed with ASD that suffered a fractured right tibia as a result of a fall. The simulations took place in an emergency department at the point of pre-discharge. To maintain consistency students read pre-simulation information before the scheduled simulation. The same faculty was responsible for orally pre-briefing students on simulation outcomes and to maintain the simulation time and flow for repeating the simulation for all student groups over the past three-year period. Two faculty were responsible for the debriefing process for all of the simulations. A forth faculty served as an SP in the role of the patient's mother.

The SP was initially educated on the specifics of a simulation, the role to be assumed, the actual simulation scenario, simulation outcomes, and the debriefing process. Subsequently, the SP was educated on any changes to the role or simulation scenario and was always debriefed by two of the four faculty again for consistency. After conducting the simulations several times the faculty wanted to better understand the simulation experience from his (SP) perspective.

\section{A STANDARDIZED PATIENTS' VIEWPOINT (IN HIS OWN WORDS)}

I had taken part in other simulations as a participant but only with a broad role. For example, I had played the role of a trauma victim during a trauma simulation. Taking part in that simulation was an interesting experience because I saw how

Published by Sciedu Press nurses, nursing students, and other health care professionals entered the room. But this particular simulation was very different because I was playing the role of the standardized patient. Specifically, I was asked to realistically portray a teenager with ASD.

When I was first asked to participate in the simulation I was excited as I was working as an Applied Behavior Analyst (ABA) therapist at a local clinic — working with children and young adults with ASD. To me it felt like validation for all I the experience that I had received in the clinical environment. In addition, I felt like I was being asked to take my knowledge to the next level - to help others learn about ASD. It also gave me a hope that I would be participating in a new opportunity, but also working with nursing professionals that wanted to bring ASD to their nursing students. It gave me a small bit of pride to know that I was working to improve the patient experience for people with ASD.

The role of the SP takes preparation. The night before the simulation, I take time to visualize, in my mind, the simulation a couple of times. This helps me get ready for the simulation experience. It puts me in the right mindset-I equate it to an actor getting ready for a role. I also think about common behaviors like stimming (e.g., rocking, flapping) that teenagers with ASD exhibit. Visualization allows me to draw on my past clinical experiences so that I can simulate these same types of behaviors while in the role of the SP. Preparation also includes reviewing the script so that I am following the simulation scenario the researchers have developed.

Because we have to run the simulations multiple times in one day, sometimes 10-15 times, I have to repeat the same behaviors over and over again. For me, this has never been a problem because it me to get deeper into the role. However, I can see that if an elderly adult had to play a SP, this could be challenging due to the physical exertion it takes.

I am in constant communication with the School of Nursing (SON) faculty. At the end of each simulation, one of the faculty will give me feedback. For example, if I am portraying too much of a specific behavior (i.e., squirming, pinching, screaming) that the students cannot get through the simulation, then faculty will give me direction on what they want for the next simulation. To me, this added feedback, allows me to adjust my portrayal.

The most satisfying part of this process for me is interacting with the nursing students themselves. Because I have now worked as a SP for several years, I can pick up on the mood (e.g., anxious, prepared) of the nursing students when they enter the room. I try to play off the student's emotions, at- 
titudes, and preparedness or lack of preparedness with still keeping in the requirements of the simulation. They may not realize it, but the students definitely play a role in what direction the simulation goes.

Playing the role of the SP and being an integral part of simulations has taught me the value of simulations as a way to learn. ASD individuals have a variety of behaviors (e.g., aggression, escape) and co-morbidities (e.g., seizures, ADHD) that would never allow these individuals to participate in such simulations. Therefore, SP's are essential. I feel like I am offering a service. In addition, it's an opportunity for nursing students to practice skills without the risk of hurting a real patient.

One consistent theme that I have noticed is that some students are anxious about interacting when they see certain types of behaviors - especially aggression or fowl language (e.g. cursing, nasty talking). It's not always comfortable for me to use such language or portray such behavior. Being accurate is important because these behaviors are what students (and nurses) will experience with certain individuals with ASD. I believe it is important for students to have a realistic experience so they are better prepared when they do encounter a person with ASD.

The biggest gain as a SP is empathizing with how healthcare professionals interact with individuals with ASD. Playing this role has highlighted to me the continued need for improved interaction between the healthcare professional and individuals with ASD. Since starting my work with this simulation study, I've gone back to school to become a nurse myself.

\section{THE FACULTY'S VIEWPOINT ON WORK- ING WITH A STANDARDIZED PATIENT}

Working with an SP can be a wonderful experience but also comes with some challenges. The following information may be helpful to faculty anticipate working with an SP and also faculty who have worked with an SP but seek new ideas on how to select and interact with the SP.

\section{Selecting The Right PERSON}

Securing the right person to assume the SP role is important. Regardless, of the type of patient the SP is assuming, the process will be greatly enhanced if the SP has previous experience or knowledge of the subject. Our SP had several years of experience working with teenagers diagnosed with ASD who provided the background information to effectively "be an teenager with ASD in an emergent setting." He knew the verbal (e.g., oral and written) and non-verbal (e.g. facial expressions, gestures, and eye contact) language that would 94 be exhibited by a teenager with ASD. In addition, he could successfully simulate ASD behaviors such as, stimming behaviors (e.g., flapping, rocking). Our SP also knew sensory triggers that would cause a reaction for a teenager with ASD. For example, individuals with ASD have sensitivities (e.g., hyper and hypo) to tactile and olfactory. During the simulation, when a nursing student, placed the blood pressure cuff onto the SP - the SP knew to react with a wail and flail because of the tightness of the cuff. Similarly, if the student nurse attempted to use rubbing alcohol or hand sanitizer, the SP knew to react to it because teenagers with ASD often have an aversion to certain smells or touch.

Their environment due to all the sensory factors that surround them affects individuals with ASD - and, emergency rooms (used in the simulation) are ridden with loud noises (e.g., alarms, overhead paging) and bright lights (e.g., fluorescent). When a nursing student walked into the simulation room and flips on the overhead light, the SP knew to react accordingly. In our simulation, he curled up into a ball and started to exhibit stimming behavior (e.g., rocking, flapping).

\section{APPRECIATE THE COMMITMENT OF THE STANDARDIZED PATIENT}

As faculty the tendency is to focus on the student's experience and the simulation outcomes. However, it is very important to appreciate the commitment the SP has undertaken including the time for preparation, the physicality of the role, and the exhaustion some may experience after the day of running multiple simulations. The SP is not just an actor or someone who simply volunteers to play a role in a simulation. The SP is vital to the simulation experience and meeting the needs of the simulation outcomes. There may be an opportunity to pay an SP for participation through grants or department funding. It is important to establish any form of payment, or lack of, during the initial meeting. If the simulation will occur over the course of several hours providing snacks, drinks or a meal may be appropriate. There should also be a period of rest for the SP to regain composure, take a bathroom break, and obtain refreshment. Serious, challenging simulation such as ASD can be exhausting physically, mentally and emotionally so a rest period should be built into the day. In addition, when scheduling the simulation consideration of the SP's work schedule, the simulation laboratory availability and student/faculty convenience is necessary. Our simulation took months to schedule.

Convey to the standardized patient the need to prepare for the role Because this simulation had many layers (e.g., presentation to the ED thru ED discharge), the commitment of the $\mathrm{SP}$ was essential. An SP must be willing to memorize what 
the objectives of the simulation are. In addition, the SP must review the script and simulation scenario prior to running the simulation. Preparation will include communicating with the SP how physically demanding a role may be. In the case of our simulation, the role would be considered physically demanding (e.g., flailing, jumping, wiggling, kicking) for an older adult - especially when the simulation lasted eight minutes and was held 10-15 times in a four-hour period. An SP may have to adjust their personal schedule in order to accommodate the demanding role- for example, adjust their sleep schedule, meal times.

\section{DEBRIEFING IS IMPORTANT}

Debriefing is required by the standards for simulation as established by the International Nursing Association for Clinical Simulation and Learning (INACSL), ${ }^{[7]}$ but from a practical standpoint debriefing is essential. We communicated with our SP after each simulation. We provided feedback as to whether the SP was exhibiting realistic behaviors of a teenager with autism. If behaviors were too intense prohibiting students from completing expected nursing interventions, we would ask the SP to tone down his behavior responses.

Debriefing as an entire simulation team was equally essential. We would meet after all the simulations were completed. We discussed how the simulations unfolded and asked a variety of questions, "What went well?" "What needs to be improved?" But, we also discussed logistical questions such as, "What type of props do we need for future simulations?" "With the physicality of the simulation do we need the room cooler?"

\section{CAPTURE THE OPPORTUNity TO LEARN AND TEACH}

We believed every opportunity was an occasion for learning by all people involved in the simulation process not just the students. Learning can, and did, take place even in situations not planned by the faculty. As our SP stated his behaviors often played off of the student's actions. If the students seemed unprepared or unsure of what to do the SP would use that conduct as an opportunity to display a specific behavior to cue the students into action. Faculty taught the SP about the simulation outcomes and discussed each simulation experience for a better or a change in performance. The SP used his expertise about ASD to teach the faculty and the students enhancing everyone's knowledge of ASD from his perspective. The students were very open and eager to share their simulation experience and how the SP positively impact the simulation had on them as they move towards transitioning to the professional registered nurse role.

\section{DiscuSSION}

Working with a SP is essential when developing a realistic simulation, especially when a mannequin will not glean the results (e.g., interaction with students) that a real person will glean. The selection of the SP must be detailed including exactly what skill set and experience the individual brings to the SP experience. In our simulation, our SP had years of working experience with the autistic population. But the same approach should be utilized regardless of the simulation (e.g., cardiac, respiratory).

Prior to securing the SP, consideration needs to be given to how to the logistics of the SP experience will unfold including things such as parking, pay, bathroom breaks, and refreshments. It has been our experience, that taking these types of details into consideration and addressing each one makes the SP feel special and wanting to work in the simulation again and again.

\section{Conclusion}

To conclude the SP is a major participant in the simulation experience for students and faculty. It is imperative that faculty secure the right person to serve in an SP role, continually educate the SP as to specific outcomes to be achieved by the students and the simulation. Time and consideration should be carefully given to securing and educating the SP. The SP should have the opportunity to express positive and negative feelings, and suggestions through a personal debriefing with faculty. Learning can take place in all situations and should be embraced by all persons involved in the simulation. Above all, demonstrate an appreciation for the time and commitment the SP is providing for student learning.

\section{REFERENCES}

[1] McIntosh CE, Thomas CM, Allen RA, et al. Using a combination of teaching and learning strategies and standardized patient for a successful Autism simulation. Clinical Simulation in Nursing. 2015; 11(3): 143-152. https://doi.org/10.1016/j.ecns .2014.11.008

[2] Webster D. Using standardized patients to teach therapeutic commu- nication in psychiatric nursing. Clinical Simulation in Nursing. 2014; 10(2): e81-86.

[3] Nehring WM. U. S. boards of nursing and the use of high-fidelity patient simulators in nursing education. Journal of Professional Nursing. 2011; 24(2): 109-117.

[4] Schinnick MA, Woo MA, Mentes JC. Human patient simulation: 
State of the science in prelicensure nursing education. Journal of Nursing Education. 2011; 50: 65-72. PMid:21210612 https: //doi.org/10.3928/01484834-20101230-01

[5] International Nursing Association for Clinical Simulation and Learning (INACSL; 2016). Clinical Simulation in Nursing. 2016; 12(12): 529-570. Available from: http://www.nursingsimulation.or g/issue/S1876-1399(16) X0012-6

[6] Lewis KL, Bohnert CA, Gammon WL, et al. The association of standardized patient educators (ASPE). Standards of best practice (SOBO). Advances in Simulation. 2017.

[7] Lopreiato JO, Downing D, Gammon W, et al. Terminology concepts working group. Healthcare Simulation Dictionary. 2016.

[8] Robinson-Smith G, Bradley P, Meakim C. Evaluating the use of standardized patients in undergraduate psychiatric nursing experiences. Clinical Simulation in Nursing. 2009; 5: e203-e211.

[9] Jefferies P. Simulation in Nursing Education: From Conception to Evaluation (2nd ed.). New York, NY: National League for Nursing. 2012.

[10] Hart JA, Chilcote DR. "Won't you be my patient?" Preparing theater students as standardized patients. Journal of Nursing Education. 2016; 55(3): 168-171. PMid:26926219 https://doi.org/10.3 928/01484834-20160216-09

[11] Jacobs AC, van Jaarsveldt DE. The character rests heavily within me: Drama students as standardized patients in mental health nursing education. Journal of Psychiatric and Mental Health Nursing. 2016; 23(3-4): 198.

[12] Benner P, Sutphen M, Leonard V, et al. Educating nurses: A call for radical transformation. San Francisco, CA: Jossey-Bass; 2010.

[13] Joan A, Kotowski P. Using standardized patients in an undergraduate nursing health assessment class. Clinical Simulation in Nursing.
2017; 13(7): 309-313. https : //doi.org/10.1016/j.ecns. 201 7.05 .003

[14] Goh YS, Selvarajan S, Chng ML, et al. Using standardized patients in enhancing undergraduate students' learning experience in mental health nursing. Nurse Education Today. 2016; 45: 167-172.

[15] Luctkar-Flude M, Wilson-Keates B, Larocque M. Evaluating highfidelity human simulators and standardized patients in an undergraduate nursing health assessment course. Nursing Education Today. 2012; 32: 448-452. PMid:21565436 https://doi .org/10.1016/ j.nedt. 2011.04.011

[16] American Psychiatric Association. Diagnostic and statistical manual of mental disorders (5th ed.). Washington DC: Author. 2013.

[17] Tuzer H, Dinc L, Elcin M. The effects of using high-fidelity simulators and standardized patients on the thorax, lung, and cardiac examination skills of undergraduate nursing students. Nurse Education Today. 2016; 45: 120-125. PMid:27449150 https ://doi.or $\mathrm{g} / 10.1016 / \mathrm{j}$. nedt .2016 .07 .002

[18] Jeffries PR, Rogers B, Adamson K. NLN Jeffries simulation theory: Brief narrative description. Nursing Education Perspectives. 2015; 36(5): 292-293.

[19] van Soeren M, Devlin-Cop B, MacMillian K, et al. Simulated intraprosessional education: An analysis of teaching and learning processes. Journal of Intraprofessional Care. 2011; 25(6): 434-440.

[20] Fenske CL, Harris MA, Aebersold ML, et al. Perception versus reality: A comparative study of the clinical judgment skills of nurses during a simulated activity. Journal of Continuing Education in Nursing. 2013; 44(9): 399-405.

[21] Kaplan BG, Abraham C, Gary R. Effects of participants' vs. observation of a simulation experience on testing outcomes implications for logistical planning for a school of nursing. International Journal of Nursing Education Scholarship. 2012; 9(1): 1-5. 